\title{
Cardiologia
}

\section{Interesse Científico em Saúde Cardiovascular e Reabilitação Cardíaca}

\author{
Sebastião David Santos-Filho \\ Professor da Universidade Severino Sombra nas Disciplinas de Biofísica \\ e Fisiologia, sdsfilhodterra.com.br
}

Resumo: a prescrição de exercícios físicos deve ser ajustada à medida que a capacitação cardiovascular e a capacidade física para as atividades diárias melhorem a partir do programa de reabilitação proposto pela fisioterapia. Verificou-se o número de publicações em revistas indexadas citadas no PubMed (http://www.ncbi.nlm.nih.gov/entrez/query.fcgi) relacionadas com a reabilitação cardíaca e aos principais exercícios utilizados no tratamento. MÉTODO: avaliou-se no indexador PubMed (10 de agosto de 2009,) o número de publicações em reabilitação cardíaca e alguns sinais e sintomas de doenças cardíacas vasculares e reabilitação cardíaca e exercícios de reabilitação. $O$ número de publicações de cada sinal/exercício foi dividido pelo total obtido para reabilitação cardíaca, calculando-se um percentual de publicações para cada sinal/exercício. RESULTADOS: Em 14705 citações verificou-se maior interesse científico em publicações sobre infarto do miocárdio, angina e hipertensão. Verificou-se também grande interesse em publicações sobre exercícios aeróbicos. CONCLUSÃO: a análise dos resultados mostra o interesse da comunidade científica pelos sinais e sintomas das doenças cardíacas. Verificou-se a importância dos exercícios aeróbicos para a reabilitação cardíaca, sugerindo uma discussão maior pelos profissionais de fisioterapia dos procedimentos de reabilitação cardiovascular.

Palavras-chaves: Reabilitação Cardíaca. Doenças Cardiovasculares. Exercícios Aeróbicos.

Rev. de Saúde, Vassouras, v. 1, n. 1, p. 33-40, jan./mar., 2010 


\title{
Scientific Interest in Cardiovascular Health and Cardiac Rehabilitation
}

\begin{abstract}
: the prescription of physical exercises must be adjusted in the proportion in that the cardiovascular capacitating and the physical capacitating for the diary activities were going better according to rehabilitation program proposed by physiotherapy. It verified the number of publications in indexed journals cited on PubMed related with the cardiac rehabilitation and the main exercises utilized in the treatment. METHOD: It evaluated in the PubMed (august 10, 2009) the number of publications in cardiac rehabilitation and some signals and symptoms of cardiovascular diseases and, cardiac rehabilitation and rehabilitation exercises. The number of publications of each signal/exercise was divided by total obtained for cardiac rehabilitation, calculated a percentile of publications for each signal/exercise. RESULTS: In 14705 citations verified high scientific interest in publications about myocardial infarct, angina and hypertension. It also verified biggest interest in publications about aerobic exercises. CONCLUSION: The analysis of the results shows the interest of the scientific community about signals and symptoms of the cardiac diseases. It verified the importance of the aerobic exercises for the cardiac rehabilitation, suggesting a discussion by professionals of physiotherapy of the procedures of cardiac rehabilitation.
\end{abstract}

Keywords: Cardiac Rehabilitatio. Cardiovascular Diseases. Aerobic Exercises.

\section{Introdução}

Nas últimas décadas, o padrão de morbimortalidade no Brasil vem passando por um período de transição. Segundo Alves e Figueiroa (2004), vem ocorrendo um melhor controle das doenças infecciosas e um predomínio das doenças crônico degenerativas. As doenças cardiovasculares correspondem a principal causa de morte no mundo e representaram 27,3\% dos óbitos registrados no Brasil no ano de 2000. Guyton e Hall (2002) descrevem que a causa mais comum de morte na sociedade ocidental é a cardiopatia isquêmica e que cerca de $40 \%$ das pessoas nos Estados Unidos da América morrem devido a essa causa.

A recuperação de um paciente que tenha sido acometido por um distúrbio cardiovascular envolve protocolos de tratamento que levam em consideração diversos parâmetros. A prescrição de exercícios físicos deve ser ajustada à medida que a capacitação cardiovascular e a capacidade física para as atividades diárias melhorem com o esquema de reabilitação proposto pela Fisioterapia. Ao prescrever exercícios físicos, o fisioterapeuta e demais profissionais de saúde envolvidos com a reabilitação procuram elaborar e programar uma quantidade ou dose específica de exercícios 
seguros e benéficos para o indivíduo tendo como meta aprimorar a função cardiovascular e, se possível, diminuir, o risco de doença cardíaca coronariana. Levando em consideração as condições físicas do indivíduo, ajustando-se, assim, sua intensidade, frequência e duração. [Carrol e Pollock, 1992; Graves e Pollock, 1993; Fardy et al., 1998; Trupp, 2004; Carré, 2009; Adams et al., 2009]

As formas mais comuns de exercícios para os pacientes cardíacos ou para aqueles interessados na função cardiovascular são as atividades aeróbicas contínuas que utilizam as extremidades superiores e inferiores. [Song, 2003; Izawa et al., 2004; Kooi et al., 2005]

A doença cardiovascular é agravada pelo aparecimento de sinais e sintomas e por fatores de risco como hipercolesterolemia, insuficiência valvar, angina, infarto do miocárdio, fumo, sedentarismo, obesidade, hipertensão, diabetes melittus, história familiar de doença coronariana e estresse [Fardy et al., 1998; Song, 2003; Alves e Figueiroa, 2004; Aronow, 2005; St Sauver et al., 2005]. Dentre todos esses o infarto do miocárdio, angina e hipertensão são provavelmente os fatores de maior importância para a comunidade científica. Um novo fator de risco para doenças cardiovasculares tem sido identificado: o baixo peso ao nascer, como reflexo da subnutrição fetal e no primeiro ano de vida. [Alves e Figueiroa, 2004; Korentz et al., 2007]

$O$ interesse da comunidade científica num assunto específico pode ser avaliado pela análise dos artigos publicados em periódicos indexados em bancos de dados confiáveis. Um desses bancos é o PubMed que inclui, em geral, os resumos dos artigos e links para muitos sites que oferecem textos completos de artigos. O PubMed tem sido utilizado por vários autores para acompanhar o número de publicações em vários temas relacionadas com as Ciências da Saúde. No estudo da diabetes tipo 1 [Manthei et al., 2004], na avaliação de riscos cardiovasculares de pacientes com espondiloartropatias [Peters et al., 2004], na revisão da prevenção do diabetes tipo II [Qvigstad, 2004], na revisão dos critérios necessários para diagnóstico precoce do melanoma cutâneo [Abbasi et al., 2004], na avaliação da fibrilação atrial induzida por drogas [van der Hoft et al., 2004], na avaliação de artigos sobre medicina tradicional chinesa e mais especificamente da técnica de moxabustão [Santos-Filho et al., 2004].

O objetivo desse trabalho foi quantificar as publicações em revistas indexadas citadas no PubMed relacionadas com a reabilitação cardíaca e aos principais exercícios utilizados no tratamento de pacientes acometidos por doença cardiovascular.

\section{Método}

Para a avaliação das publicações pertinentes foi utilizado o indexador PubMed (www.ncbi.nlm.nih.gov/PubMed/) no dia 10 de agosto de 2009. O número de publicações em reabilitação cardíaca e alguns sinais e sintomas de doenças cardíacas foram selecionados para análise.

Após a identificação do número de publicações em reabilitação cardíaca (cardiac rehabilitation) foi realizada outra análise, agora com esse termo e as palavras hipertensão (hypertension), diabetes mellitus (diabetes mellitus), obesidade (obesity), angina (angina), infarto do miocárdio (myocardial infarction), insuficiência da valva 
mitral (mitral valve), e da valva tricúspide (tricuspid valve). O número de publicações em cada uma das palavras citadas e pesquisadas foi dividido pelo total obtido para reabilitação cardíaca, calculando-se um percentual de publicações para cada uma.

Nessa etapa do trabalho foi realizado o estudo usando-se a reabilitação cardíaca com os exercícios e procedimentos relacionados, empregando-se as palavras bicicleta ergométrica (Bycicle ergometry), treinamento de resistência (endurance training), exercício aeróbico (aerobic exercise), exercício de estresse (stress exercise), exercício em esteira (treadmill exercise), treinamento de força (strength training) e exercícios anaeróbicos (anaerobic exercises).

O número de publicações em cada uma das palavras usadas para os exercícios foi dividido pelo total obtido para reabilitação cardíaca, calculando-se um percentual de publicações para cada um desses exercícios.

\section{Resultados}

Quando foi analisamos o número de citações em reabilitação cardíaca no PubMed foram identificamos 14705 .

O cálculo do percentual das publicações que envolve reabilitação cardíaca e sintomas ou sinais associados com doenças cardíacas, como hipertensão, angina, infarto do miocárdio, diabetes mellitus, obesidade, insuficiência da valva mitral, valva tricúspide revelou que foi identificado um valor bem superior para o infarto do miocárdio do que para os demais como mostrados na Tabela I.

$\mathrm{Na}$ tabela II pode-se identificar percentagens de publicações, quando se relacionou reabilitação cardíaca e diversos procedimentos que potencialmente seriam sendo utilizados em pacientes que sofreram algum problema cardiovascular. Verificou-se que esse percentual de publicações, segundo essa metodologia, é bem maior para os exercícios aeróbicos seguidos dos exercícios de estresse (Tabela II).

Tabela I - Interesse da comunidade científica em diferentes sinas e sintomas de doenças cardiovasculares e reabilitação cardíaca.

\begin{tabular}{lc}
\hline Cardiopatia & \% de publicações \\
\hline Insuficiência da valva tricúspide & 0,26 \\
Obesidade & 2,76 \\
Insuficiência da valva Mitral & 1,53 \\
Diabetes Mellitus & 1,63 \\
Hipertensão & 7,88 \\
Angina & 5,56 \\
Infarto do miocárdio & 20,13 \\
\hline
\end{tabular}

Rev. de Saúde, Vassouras, v. 1, n. 1, p. 33-40, jan./mar., 2010 


\section{Tabela II - Interesse da comunidade científica em exercícios de reabilitação cardíaca.}

\begin{tabular}{lc}
\hline Exercício & \% de publicações \\
\hline Treinamento de força & 3,56 \\
Exercícios de estresse & 4,30 \\
Exercícios de resistência & 3,46 \\
Exercícios anaeróbicos & 1,81 \\
Exercícios aeróbicos & 42,43 \\
\hline
\end{tabular}

\section{Discussão}

A informação científica é de grande valor para a tomada de decisões e na área de Saúde isso tem particular importância. É altamente sugestivo supor que o aproveitamento do conhecimento científico também tem contribuído para a modificação do padrão de morbimortalidade no Brasil e no mundo. Esse fato teria possibilitado um melhor controle das doenças infecciosas e ajudado no aprimoramento de métodos de diagnóstico e tratamento de doenças crônico-degenerativas.

As doenças cardiovasculares correspondem à principal causa de morte no mundo e representaram 27,3\% dos óbitos registrados no Brasil no ano de 2000 [Alves e Figueiroa, 2004] e a cardiopatia isquêmica é responsável por cerca de $40 \%$ das mortes de pessoas nos Estados Unidos da América [Guyton e Hall, 2002]. A recuperação de um paciente que tenha sido acometido por um distúrbio cardiovascular envolve protocolos de tratamento que levam em consideração diversos parâmetros [Carrol e Pollock, 1992; Graves e Pollock, 1993; Fardy et al., 1998; Izawa et al., 2004; Kreizman e Allen, 2005; Carré, 2009].

A identificação das investigações sobre sinais e sintomas relacionados com os distúrbios cardiovasculares poderia ser relevante no estabelecimento de condições e programas que poderiam minimizar o surgimento ou o avanço do número de casos. $\mathrm{O}$ Pubmed é um indexador científico largamente empregado em publicações por diversos autores [Manthei et al., 2004; Peters et al., 2004; Qvigstad, 2004; Abbasi et al., 2004; van der Hooft et al.; 2004; Santos-Filho et al., 2004]. Nossos resultados revelam que ao usar a metodologia citada, o infarto de miocárdio, quando associado com a reabilitação cardíaca, é o sintoma indicativo de doença cardiovascular mais citado em comparação com hipertensão, angina, infarto do miocárdio, diabetes mellitus, ou obesidade, insuficiência da valva mitral, valva tricúspide (Tabela I).

A manutenção de um programa de exercícios poderia ser um dos fatores que contribuiriam para promover qualidade de vida relacionada com a saúde e um nível de atividade física compatível com a doença cardíaca [Izawa et al., 2004; Adams et al., 2009].

Programas de exercícios aeróbicos poderiam maximizar as funções musculares e cardiorespiratórias e prevenir a atrofia de desuso adicional em pacientes com doenças 
musculares e cardíacas [Kooi et al., 2005]. Os resultados obtidos sobre o interesse da comunidade científica em publicar artigos sobre reabilitação cardíaca indicam o elevado interesse na realização de investigação científica sobre exercícios aeróbicos (Tabela II).

\section{Conclusão}

A análise dos resultados mostra o interesse da comunidade científica em desenvolver trabalhos sobre os sinais e sintomas das doenças cardíacas, com particular interesse no infarto do miocárdio. Mais ainda: verificou-se a importância dos exercícios aeróbicos para a reabilitação cardíaca, para o que sugere-se uma discussão maior pelos profissionais de Fisioterapia dos procedimentos de reabilitação cardiovascular. 


\section{Referências}

Abbasi, N.R., Shaw, H.M., Rigel, D.S., Friedman, R.J., Mccarthy, W.H., Osman, I., Kopf, A.W. and Polsky, D. (2004). Early diagnosis of cutaneous melanoma: revisiting the ABCD criteria. JAMA. 292, 2771-2776.

Adams, J., Roberts, J., Simms, K., Cheng, D., Hartman, J., Bartlett, C. (2009). Measurement of functional capacity requirements to aid in development of an occupation-specific rehabilitation training program to help firefighters with cardiac disease safely return to work. Am J Cardiol. 103,762-765.

Alves, J.G.B. and Figueiroa, J.N. (2002). Mortalidade infantil no Brasil e óbitos, na mesma geração, por infarto agudo do miocárdio. Cad. Saúde Pública. 20, 15251530 .

Aronow, W.S. (2005). Management of peripheral arterial disease. Cardiol Rev. $13,61-68$.

Carré, F. (2009). Is individual risk related to physical activity predictable in cardiac patient? Presse Med. 38,953-957.

Carroll, J.F. and Pollock, M.L. (1992). Rehabilitation and life-style modification in the eldery. Cardiovasc Clin. 22, 209-227.

Graves, J.E. and Pollock, M.L. (1993). Exercise testing in cardiac rehabilitation. Role in prescribing exercise. Cardiol Clin. 11, 253-266.

Guyton, A.C. and Hall, J.E. (2002) Tratado de Fisiologia Médica. 10 a ed., Rio de Janeiro: Guanabara Koogan.

Fardy, P.S., Yanowitz, F.G. and Wilson, P.K. (1998). Reabilitação cardiovascular. Aptidão física do adulto e teste de esforço. Rio de Janeiro: Revinter.

Izawa, K.P., Yamada, S., Oka, K., Watanabe, S. Omiya, K., Tijima, S., Hirano, Y., Kobayashi, T., Kasahara, Y., Samejima, H. and Osada, N. (2004). Long-term exercise maintenance, physical activity, and health-related quality of life after cardiac rehabilitation. Am J Phys Med Rehabil. 83, 884-892.

Kooi, E., Lindeman, E. and Riphagen, I. (2005). Strength training and aerobic exercise training for muscle disease. Cochrane Database Syst. Rev. 25, CD003907.

Kreizman, I.J. and Allen, D. (2005). Aging with cardiopulmonary disease: the rehab perspective. Phys Med Rehabil Clin N Am. 16, 261-265.

Manthei, E.R., Siminerio, L.M., Conley, Y., Charron-Prochownik, D., Feathers, A.S., Charles, B. and Dorman, J.S. (2004). Genetics and type 1 diabetes: online resources for diabetes educators. Diabetes Educ. 30, 961-971.

Peters, M.J., Van Der Horst-Bruinsma, L.E., Dijkmans, B.A. and Nurmohamed, M.T. (2004). Cardiovascular risk profile of patients with spondylarthropathies, particularly ankylosing spondylitis and psoriatic arthritis. Semin Arthritis Rheum. 34, 585-592. 
PubMed http://www.ncbi.nlm.nih.gov/entrez/query.fcgi, National Library of Medicine, acesso em 10/08/2009.

Qvigstad E. (2004). Prevention of type 2 diabetes--an overview. Tidsskr Nor Laegeforen. 124, 3047-3050.

Santos-Filho, S.D., Bastos, S.R.C., Pereira, F.A.O., Senna-Fernandes, V., França, D., Guilhon, S. and Bernardo-Filho, M. (2004). Tradicional medicine: an evaluation of the interest of the publication of scientific papers about moxibustion. Journal of Medical Sciences. 4, 59-62.

Song, K.J. (2003). The effects of self-efficacy promoting cardiac rehabilitation program on self-efficacy, health behavior and quality of life. Tashan Kanho Hakhoe Chi. 33, 510-518.

St Sauver, J.L., Hagen, P.T., Cha, S.S., Bagniewski, S.M., Mandrekar, J.N., Curos, A.M., Rodehefter, R.J., Roger, V.L. and Jacobsen, S.J. (2005). Agreement between patient reports of cardiovascular diseases and patient medical records. Mayo Clin Proc. 80, 203-210.

Trupp, R.J. (2004). Cardiac resynchronization therapy: optimizing the device, optimizing the patient. J Cardiovasc Nurs. 19, 223-233.

Van der Hooft, C.S., Heeringa, J., Van Herpen, G., Kors, J.A., Kingma, J.H. and Stricker, B.H. (2004). Drug-induced atrial fibrillation. J Am Coll Cardiol. 44, 2117-2124. 